The following paper posted here is not the official IEEE published version. The final published version of this paper can be found in the

Proceedings of IEMDC'07 (2007 : Antalya, Turkey):pp.196-201

Copyright (C) 2007 IEEE.

Personal use of this material is permitted. However, permission to reprint/republish this material for advertising or promotional purposes or for creating new collective works for resale or redistribution to servers or lists, or to reuse any copyrighted component of this work in other works must be obtained from the IEEE. 


\title{
Detection of Broken Rotor Bar Faults and Effects of Loading in Induction Motors during Rundown
}

\author{
Randy Supangat ${ }^{1}$, Jason Grieger, Nesimi Ertugrul, Wen L. Soong, Douglas A. Gray, and Colin Hansen ${ }^{*}$ \\ School of Electrical and Electronic Engineering and ${ }^{*}$ School of Mechanical Engineering \\ University of Adelaide \\ Australia \\ Irsupang@eleceng.adelaide.edu.au
}

\begin{abstract}
The detection of broken rotor bar faults based on the common steady-state Fourier transform technique is known to be dependent on the loading condition and the quality of the supply. This paper attempts to minimise these issues by utilising the induced voltage in the stator windings after supply disconnection. When the supply is disconnected, the stator current rapidly drops to zero and the only source of the stator induced voltage an instant after the supply disconnection is due to currents in the rotor. The rotor currents are sensitive to broken rotor bar faults and directly affect the rundown induced voltage in the stator windings. The performance of two different broken rotor bar detection techniques, based on the Fourier transform and the wavelet transform, are investigated over a wide range of loading conditions.
\end{abstract}

\section{INTRODUCTION}

The broken rotor bar (BRB) fault is one of the most commonly investigated faults in induction motors. Broken rotor bar faults are usually detected by utilising the Fourier transform (FT) of the steady-state stator current [1]-[3]. The techniques usually measure the broken rotor bar sideband (1) magnitudes, where the magnitudes of the faulty motor are higher than the magnitudes of the healthy motor. It is also found that the greater the severity of the fault, the higher the magnitudes of the broken rotor bar sidebands. However, the magnitudes of these sidebands are sensitive to the level of load and to supply harmonics. For example, the broken rotor bar fault is more difficult to detect when the load is low and the quality of the supply is poor (i.e. contains a lot of time harmonics).

$$
f_{B R B}=f(1 \pm 2 s)
$$

where $f_{B R B}$ is the broken rotor bar sidebands, $f$ is the supply frequency, and $s$ is the slip.

One alternative to reduce the effect of load is to utilise the starting current. The starting current is typically 5 to 6 times the rated current and is not sensitive to the level of load. Also during starting, the rotor currents are high and so rotor faults should be more evident than under steady-state operation.

Investigations involving detecting broken rotor bar faults using the starting current have been reported previously in [4][6]. The work in [4] described how the envelope of the starting current can be used to differentiate between healthy and faulty motors by means of wavelet indicators. It was shown that the greater the severity of the broken rotor bar fault, the higher the wavelet indicator. The investigation in [5] started by utilising an adaptive signal cancellation technique to remove the fundamental component of the starting current. The residual current was then processed through a discrete wavelet transform, where the wavelet coefficients were used to differentiate between healthy and faulty motors. The work in [6] used the wavelet transform on the Hilbert transform of the starting current signal, where the resultant wavelet coefficient was then processed to compute what was called the wavelet ridge. The wavelet ridge was shown to be able to differentiate healthy and faulty motors.

Another alternative to minimise the potential problems in detecting a broken rotor bar fault is to utilise the induced voltage in the stator windings after supply disconnection. When the supply is disconnected, the stator current rapidly drops to zero and the only source of the stator induced voltage an instant after the supply disconnection is due to currents in the rotor. The rotor currents are sensitive to broken rotor bar faults and directly affect the rundown induced voltage in the stator windings. In particular, a broken rotor bar can cause the rotor current distribution to become distorted, and hence produce characteristic harmonics in the induced voltage.

Broken rotor bar detection using the induced voltage during rundown has been reported previously in [7] and [8]. The work in [7] was based on a $3 \mathrm{hp}, 3$-phase, four-pole induction motor under a particular load and with a particular broken bar fault. It was shown that significant differences between the amplitudes of the harmonics of healthy and faulty motors can be observed in the first 5 cycles (after disconnecting the supply) of the induced voltage. It was reported that the amplitudes of the $35^{\text {th }}$ and $37^{\text {th }}$ harmonics show the most differences, where the magnitudes of the faulty motor are significantly higher than the magnitudes of the healthy motor. However, the technique has not been investigated under different severities of broken bar fault, different motor sizes, and different loading conditions. Similarly, the work in [8] investigated the harmonics of the rundown induced voltage by utilising MUSIC (Multiple Signal Classification). It was shown that this technique is more effective than the FFT and is not affected by the loading conditions.

This paper aims to investigate the limitations and effectiveness of broken rotor bar detection using the harmonics of the induced voltage after supply disconnection under multiple loading conditions and different severities of broken rotor bar fault. In addition, the paper also proposes another method for detecting broken rotor bar faults during rundown by utilising wavelet analysis to detect the change in 
the motor output torque for a given value of slip.

\section{DESCRIPTION OF BROKEN ROTOR BAR DETECTION TECHNIQUES DURING RUNDOWN}

When an induction machine is running, the stator currents produce a constant magnitude, sinusoidally distributed magnetic field which rotates at synchronous speed. This rotating magnetic field induces a rotor current distribution which rotates with respect to the rotor at the slip frequency.

After supply disconnection, the stator currents drop to zero almost instantaneously and currents are induced in the rotor to attempt to maintain the magnetic field in the machine. The new rotor current distribution does not rotate with respect to the rotor and hence the speed of the rotating field changes from synchronous speed before supply disconnection, to the rotor speed, just after disconnection.

The rotor bar currents, an instant after disconnecting the supply, have a sinusoidal distribution when all the rotor bars are healthy [7]. When some of the rotor bars are broken, the rotor bar current distribution is no longer sinusoidal and has discontinuities at the broken bar. These rotor bar current discontinuities produces harmonics in the rundown induced voltage which can be detected.

After the supply disconnection, the induction machine is similar to a wound-field synchronous machine with a decaying field current. The induced back-emf in each stator phase winding of the induction machine is the product of three terms: the magnetic flux linkage produced by the rotor currents, the rotor speed, and a constant magnitude sinusoidal function of rotor position.

The initial value of the rotor magnetic flux linkage (and hence induced voltage) depends on the coupling between the rotor and the stator magnetic field at the instant of supply
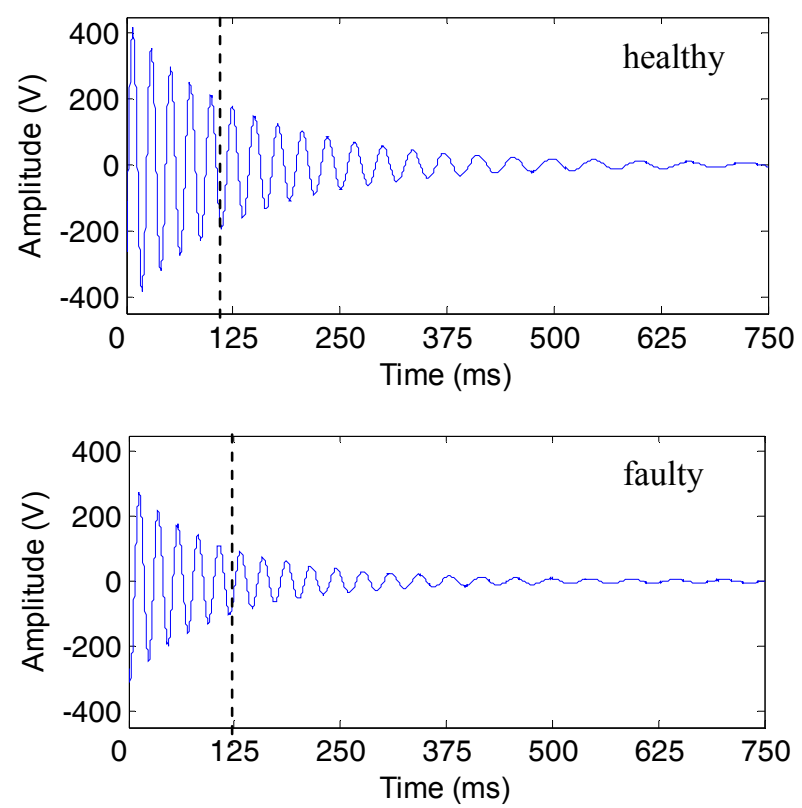

Fig. 1 - Rundown induced voltage on healthy (top) and faulty (bottom) motors when operating at rated slip before the supply disconnection. disconnection, and so can be affected by broken rotor bars. The decay of the rotor magnetic flux linkage is dependent on the rotor inductance and the rotor resistance. Broken bar faults are likely to increase the rotor resistance and hence the rate of decay of the magnetic flux linkage.

The rotor speed falls at a rate dependent on the system inertia and the load torque. The deceleration rate can thus be used to estimate the load torque. It should be noted that the output torque of an induction machine for a given value of slip reduces as the broken bar fault severity increases. This can be detected by comparing the slip before supply disconnection with the load torque estimated during rundown.

The above discussion has thus highlighted four possible methods for detecting broken rotor bar faults using the backemf voltage after supply disconnection. In summary, these are:

- harmonics in the induced back-emf voltage,

- the initial value of the rotor magnetic flux-linkage and hence back-emf voltage after supply disconnection,

- the rate of decay of the rotor magnetic flux-linkage,

- the rate of decay of the rotor speed for given operating slip before supply disconnection.

Typical measured rundown induced voltages on healthy and faulty (4 broken bars) $2.2 \mathrm{~kW}$ motors running at full-load are shown in Fig. 1. Perhaps the most obvious difference is the change in the initial rotor magnet flux-linkage value. The other effects may be present but are not easily determined by observation from the figure.

In this paper, two methods for detecting broken bars using the run-down back-emf voltage will be used. Firstly, Fourier transform analysis will be used to examine the back-emf harmonics. Secondly, wavelet analysis will be used to measure the decay rate of the rotor speed and so correlate the load (and hence motor) torque as a function of slip.

\section{A. Fourier Transform Analysis of Back-EMF Harmonics}

One way to monitor these harmonics in the run-down backemf waveform is by utilising the Fourier transform (FT). The FT can extract the frequency information of a signal and hence monitor the harmonics in the induced voltage. However, the FT requires the signal to be in the steady state for an accurate frequency interpretation and its resolution depends on the observation time, where the longer the observation time is, the higher the frequency resolution.

The rundown induced voltage is not in the steady state and so it cannot be observed for a long time. As a consequence, the rundown induced voltage needs to be processed to make it more suitable for FT analysis. One solution is to apply the FT to only the first five cycles of the induced voltage after disconnecting the supply (see the dashed line in Fig. 1) to reduce the frequency variation over the observed signal [7]. However, this process also reduces the observation time and hence reduces the frequency resolution.

\section{B. Wavelet Transform Method for Speed Decay Measurement}

The rundown induced voltage is a transient signal which 
decays at a rapid rate. As a consequence, the Fourier transform, which can only display the frequency information of a signal by using a fixed-size window, may not be the most suitable technique. In order to overcome these transient issues, the wavelet transform (2) is used. This can have provide both scale (which is related to frequency) and time information of the signal by using a variable-sized window.

Equation (2) shows that the main concept of the wavelet transform is to divide a signal into its various scaled and shifted versions of the wavelet function, in a similar fashion that the Fourier transform of a real variable divides a signal into sinusoidal components of different frequencies, magnitudes, and phases. A high scale wavelet corresponds to a "stretched" wavelet, which has a slow rate of change and hence low frequency. On the other hand, a low scale wavelet corresponds to a "compressed" wavelet with rapidly changing detail and hence high frequency. The wavelet function used for the analysis is "sym3" from the Symlet family. The "sym3" wavelet is chosen because it can highlight the broken rotor bar features found in the experimental results.

$$
C(a, \phi)=\int_{-\infty}^{\infty} f(t) \Psi(a, \phi, t) d t=\frac{1}{\sqrt{a}} \int_{-\infty}^{\infty} f(t) \Psi\left(\frac{t-\phi}{a}\right) d t
$$

where $C$ is the wavelet transform, $a$ is the wavelet scale, $\phi$ is the wavelet position, $f(t)$ is the signal, and $\Psi$ is the wavelet function [9].

It was shown earlier that broken rotor bar faults will decrease the machine output torque for a given value of slip. This will mean for the same value of slip, the load torque will be lower with a broken bar fault, and hence the rotor will take longer to slow down.

As the machine is acting as a synchronous machine after the supply is disconnected, the frequency of the induced voltage is directly proportional to the rotor speed. The wavelet transform is used to track the fundamental frequency and hence rotor speed as a function of time.

Let $v_{r d}(t)$ be the rundown induced voltage in the stator winding (3). This time representation of the induced voltage will be used to determine the number of samples that the induced voltage needs to reach a fixed decay threshold (4). The wavelet transform of the induced voltage is then used in order to extract the relevant scale (i.e. frequency) and time information of the signal (5). The resultant wavelet coefficients are then processed to determine the wavelet scale of the component with the maximum value, which should be the fundamental component, at the beginning of the signal and at the end of the signal. The difference between the wavelet scales of the peak at the beginning and at the end of observation interval is proportional to the change in the fundamental frequency and hence the speed. This change in the wavelet scale, $\Delta a$, will be used as a feature for detecting broken rotor bar faults (6).

$$
v_{r d}(t)=\alpha(t) \sin \left(\omega(t) n t_{s}\right)
$$

$$
\begin{gathered}
n_{d}=\frac{\sin ^{-1}\left(\frac{\alpha_{\max } \times \operatorname{threshold}(\%)}{\alpha(t)}\right)}{\omega(t) \cdot t_{s}} \\
C_{i_{r d}}(a, \phi)=\int_{1}^{n t_{s}} v_{r d} \cdot \Psi(a, \phi, t) d t \\
\Delta a=a\left(\max C_{i_{r d}}, \phi_{\text {start }}\right)-a\left(\max C_{i_{r d}}, \phi_{n_{d}}\right)
\end{gathered}
$$

where $\alpha(t)$ is the time-varying induced voltage amplitude, $\omega(t)$ is the time varying frequency, $n$ is the number of samples and $t_{s}$ is the sampling frequency.

\section{EXPERIMENTAL RESULTS AND ANALYSIS}

\section{A. Test Setup}

The experimental results were obtained using a purposely built test rig based on a high-speed data acquisition system (2 $\mathrm{x}$ DAQPAD-6052E boards from National Instruments, capable of simultaneous sampling) with custom-written LabVIEW software. A number of identical $2.2 \mathrm{~kW}, 415 \mathrm{~V}, 4$ pole, 32 rotor bar, star-connected induction motors were used in this test (Fig. 2). The motors were loaded by a separatelyexcited $5 \mathrm{~kW}$ DC generator. The motors were installed on the test rig using a precision laser alignment tool (Optalign Plus from Prüftechnik) and a torque wrench (Norbar) in order to ensure the accuracy and the repeatability of the data obtained. During the test, stator current and voltage sensors were attached to the motor and the sensor outputs were sampled simultaneously at $8 \mathrm{kHz}$ via an anti-aliasing filter, which was an 8th order Butterworth filter, with a cut-off frequency of 2 $\mathrm{kHz}$.

Experimental data of the motor run-down induced voltage was collected using a healthy motor and faulty motors with different levels of broken rotor bar (BRB) fault, i.e. 1 BRB, 2 $\mathrm{BRB}$, and $4 \mathrm{BRB}$. The profile of the rotor bars in the test machine can be seen in Fig. 2 (right), where a section of the rotor end-ring has been cut completely away. The broken rotor bar faults were simulated by cutting a narrow slot through the end-ring next to the lamination stack using a small diameter milling cutter to break the electrical connection between the rotor bar and end-ring. This method is used to minimise the disturbance to the rotor end-ring currents which
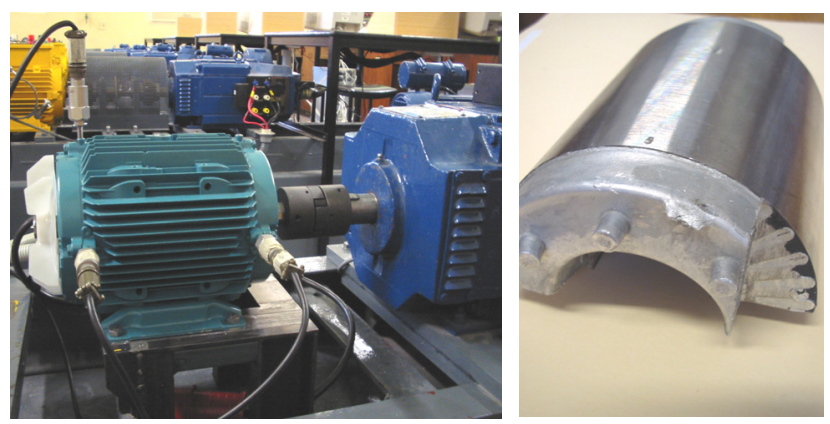

Fig. 2 - Photographs of the motor test setup (left) and the rotor crosssection showing the profile of the rotor bars (right). 
would occur if more of the end-ring was removed.

A separately-excited DC machine with a resistive load was used to load the test machine. This produced a load torque which varied approximately linearly with motor speed. For example, when the machine was disconnected from the supply at full load, the load torque also decays at a rate proportional to the motor speed. The $\%$ of rated slip given in the figures was calculated using the slip of the machine during steadystate operation.

\section{B. Fourier Transform Results and Analysis}

The power spectrum of the first five cycles of the rundown induced voltage of the healthy motor running at no load and at rated slip (full load) are shown in Fig. 3. The figure reveals that there are seven strong components in the rundown induced voltage. These 7 components are identified to be the $5^{\text {th }}, 11^{\text {th }}, 13^{\text {th }}, 17^{\text {th }}, 19^{\text {th }}, 35^{\text {th }}$, and $37^{\text {th }}$ harmonics of the fundamental. In addition, the $15^{\text {th }}$ harmonic is found to be strong in the faulty motor but not in the healthy motor. These harmonics are not exactly integer multiple of the fundamental because the rundown induced voltage is a transient signal, where the frequency of the signal components are changing over time. These eight harmonics are utilised as the broken rotor bar features for comparing the healthy and faulty motors.

Further examination of Fig. 3 shows that the widths of the harmonic peaks are changing with the load. The higher the load, the wider is the width of the harmonic peaks. This is because the rotor speed, and hence induced voltage, decays faster as the load increases, which means the frequency of the signal components are changing at a faster rate when the load is higher. In this condition, the FT averages the changes in frequency and this results in the wider peak width. The widening of the peak width reduces the frequency resolution. Therefore, the FT method is more suitable on low load cases,
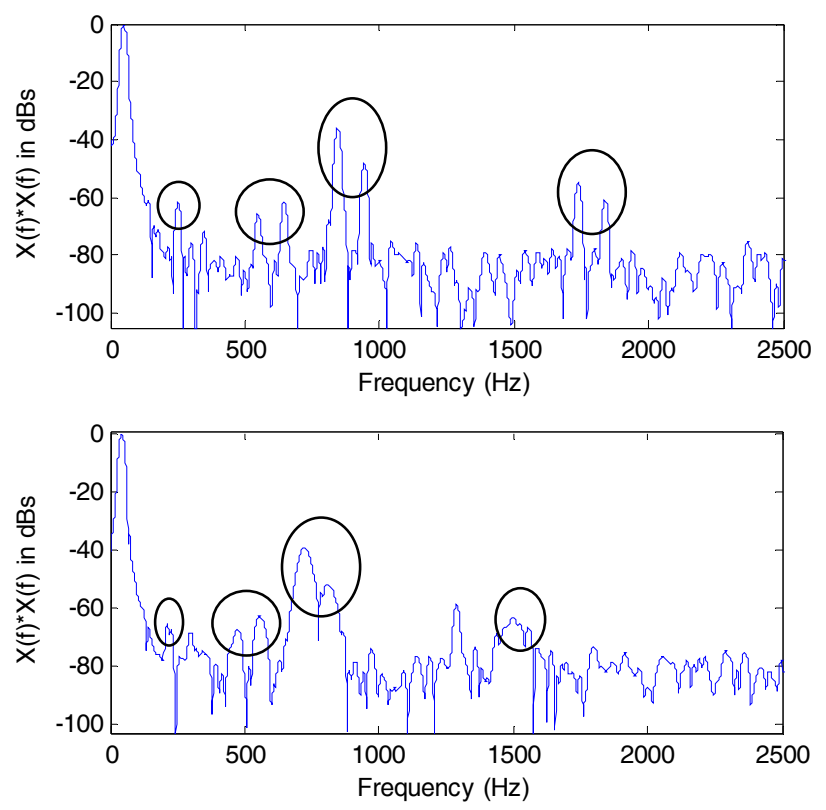

Fig. 3 - Power spectrum of the rundown induced voltage of a $2.2 \mathrm{~kW}$ healthy motor running at no load (top) and at rated slip (bottom). where the rundown induced voltage has a smaller frequency change with time.

Fig. 4 presents comparisons of four broken rotor bar harmonics for the healthy and faulty motors under different loading conditions. These harmonics are expected to be larger in the faulty motors than in the healthy motor because of the discontinuities in the rotor current distribution. In addition, they are expected to increase as the severity of the fault increases. Note that the peak detection algorithm will set the magnitude to $-100 \mathrm{~dB}$ when it cannot detect any components at the specific harmonic, as shown in the $15^{\text {th }}$ harmonics case.

Fig. 4 shows that the $5^{\text {th }}$ harmonic of the faulty motors is stronger than the same harmonic of the healthy motor under all loading conditions. However, the harmonic behaviour with
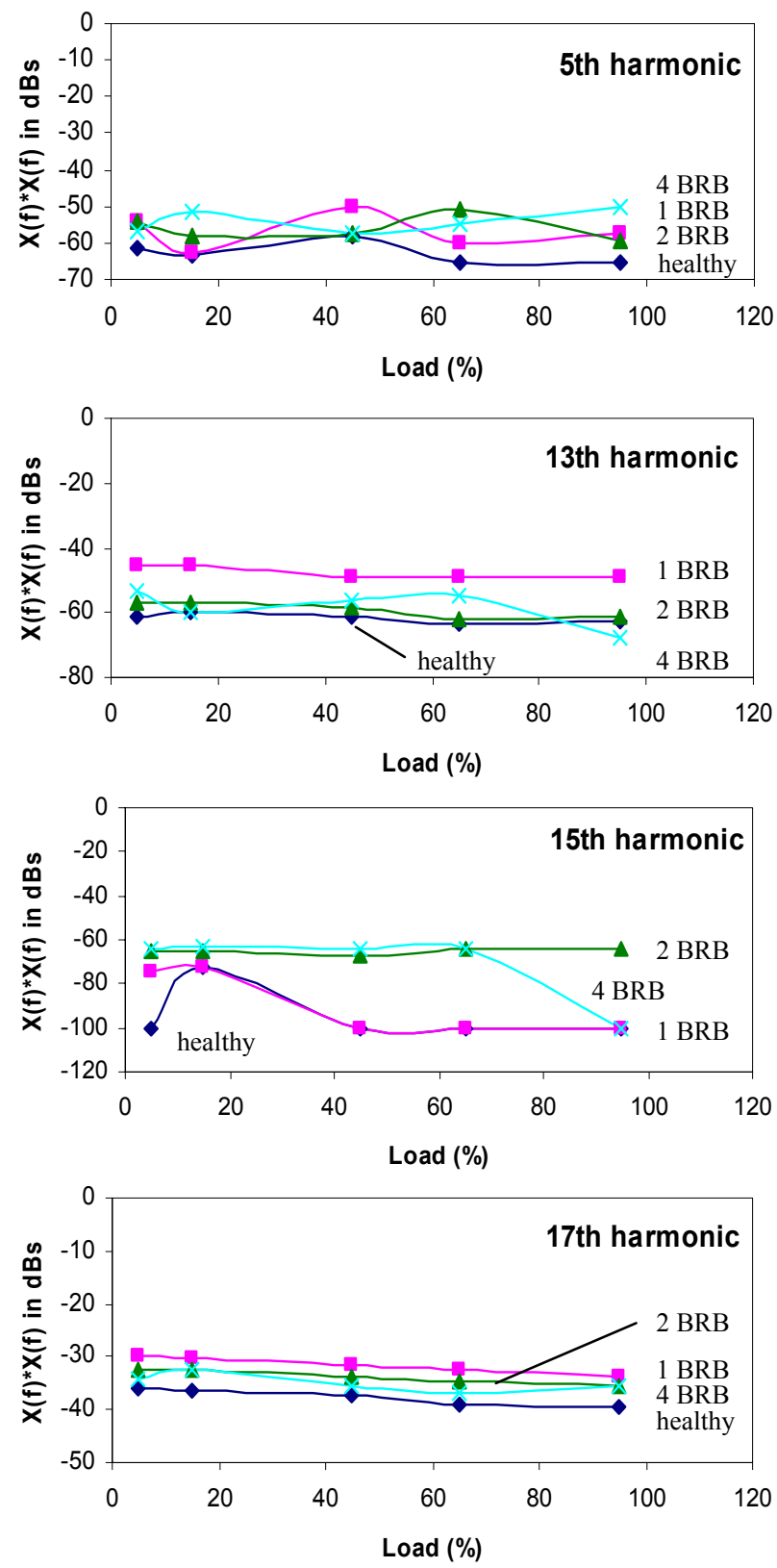

Fig. 4 - Harmonic comparisons of the different fault severities under multiple loading conditions. 
increasing fault severity, where the magnitude should increase with increasing fault severity, is not always as expected. For example, the $5^{\text {th }}$ harmonics of $1 B R B$ and $2 B R B$ are slightly larger than the $5^{\text {th }}$ harmonic of $4 B R B$ at $5 \%$ load, while the $5^{\text {th }}$ harmonic of $4 B R B$ is in fact stronger than the $5^{\text {th }}$ harmonics of $2 B R B$ and $1 B R B$ at $15 \%$ load. In addition, the $5^{\text {th }}$ harmonics have no consistent pattern with the different loading conditions. For example, the $5^{\text {th }}$ harmonic of $1 B R B=-54.1 \mathrm{~dB}$ at $5 \%$ load, then it goes down to $-63 \mathrm{~dB}$ at $15 \%$ load, and it increases again to $-50 \mathrm{~dB}$ at $45 \%$ load. Similar inconsistency can be observed for the other fault severities.

The $13^{\text {th }}$ harmonic shows very similar characteristics as the $5^{\text {th }}$ harmonic.

The $17^{\text {th }}$ harmonic is also found to be consistently stronger in the faulty motors than in the healthy motor under all loading conditions. Unlike the $5^{\text {th }}$ harmonic, the $17^{\text {th }}$ harmonic is found to have a more consistent pattern, where the harmonics only fluctuate by $\pm 2 \mathrm{~dB}$, across the different loading conditions. However, the harmonic behaviour with increasing fault severity is not always as expected, as in the case for the $5^{\text {th }}$ harmonic.

The $15^{\text {th }}$ harmonics are more evident in the faulty motor, especially the $2 B R B$ and $4 B R B$ motors, than in the healthy motor. However, the $15^{\text {th }}$ harmonic is not always present in all faulty motor cases and the harmonic behaviour with increasing fault severity is not consistent.

The rest of the broken rotor bar features (i.e. $11^{\text {th }}, 19^{\text {th }}, 35^{\text {th }}$, $37^{\text {th }}$ harmonics) are found to be inconsistent without any patterns, where the harmonics of the faulty motors can either be stronger or weaker than the healthy motor.

In summary, the Fourier transform method is able to distinguish between the healthy and faulty motors by monitoring the harmonics of the first five cycles of the induced voltage. The $5^{\text {th }}, 13^{\text {th }}$, and $17^{\text {th }}$ harmonics are found to be the more reliable features but their ability to distinguish different fault severities is limited due to the inconsistent magnitude pattern. Furthermore, the reliability of the method decreases as the load increases because the frequency resolution gets poorer as the load increases.

\section{Wavelet Transform Results and Analysis}

Typical wavelet plots of the rundown induced voltage of healthy and faulty motors running at no load and at rated slip are shown in Fig. 5 and Fig. 6 respectively. The figures show the frequency change with time of the fundamental component, which is the component between the dashed lines. This frequency change was found using the technique described in section II. $B$ and the results are depicted in Fig. 7 and Fig. 8.

As indicated in section II, broken bar faults reduce the motor torque and hence load torque for a given value of slip. Thus for a given value of slip, the rate at which the speed decays will decrease as the fault severity increases. Fig. 7 shows how the change in wavelet scale, $\Delta a$, (that is, the change in speed) over a given time interval, for the same initial value of slip, varies depending on the level of broken bar fault. This uses the same data as used in the FT analysis.
Fig. 7 shows that the change in wavelet scale $\Delta a$ tends to increase proportionally with the slip (and hence load torque). The figure also shows that $\Delta a$ tends to decrease as the severity of the broken rotor bar fault increases.

The feature, $\Delta a$, can be used to distinguish and separate the different levels of broken rotor bar fault, and hence $\Delta a$ can be considered as a useful broken rotor bar feature, especially at mid to higher loads.

Fig. 8 shows measurements taken for more load levels than Fig. 7. It shows similar trends.

It should be noted that while this technique is promising, it does have some significant limitations when applied to practical systems. Firstly, it requires tests be carried out with the motor in the healthy state to develop a baseline response curve of deceleration rate versus slip. Secondly, as it relies on
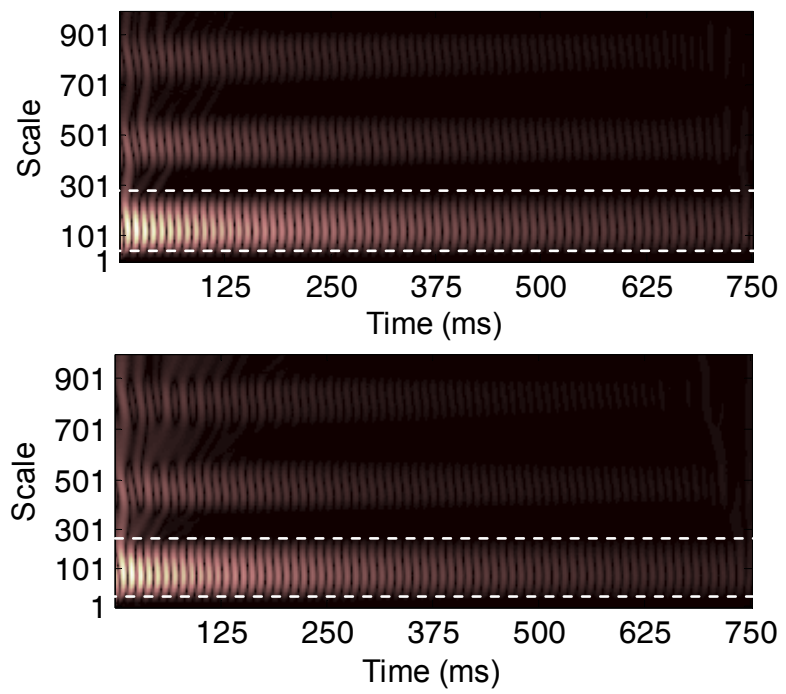

Fig. 5 - Wavelet plots of the induced rundown voltage on the healthy motor (top) and faulty motors with 4 BRB (bottom) running at no load.
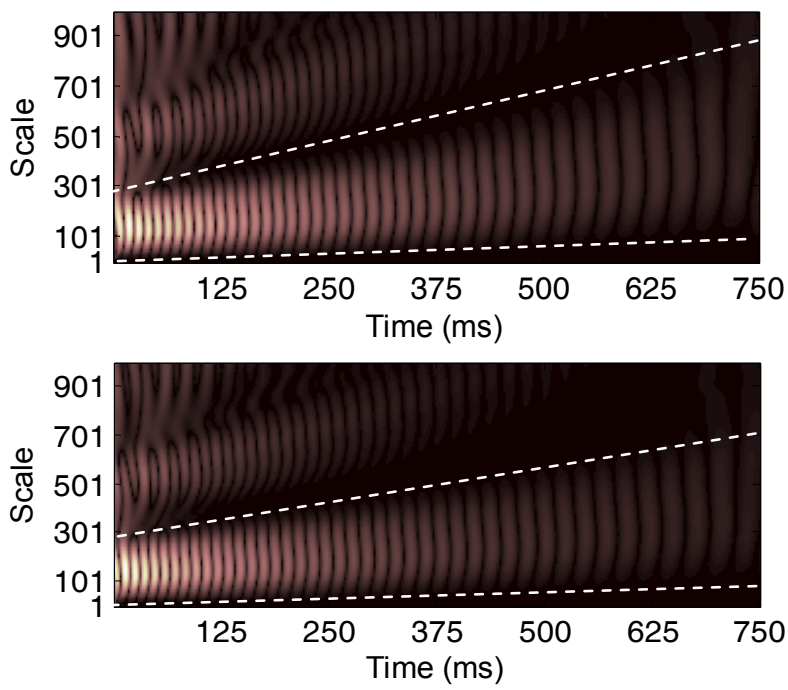

Fig. 6 - Wavelet plots of the induced rundown voltage on the healthy motor (top) and faulty motors with 4 BRB (bottom) running at rated slip. 
estimating the motor torque based on the deceleration rate after supply disconnection, and then correlating changes in motor torque with the broken bar fault severity, the approach is sensitive to changes in system inertia, rotor temperature and supply voltage. It is also sensitive to rapid fluctuations in load, as it inherently assumes that the load does not change between the steady-state operation just before supply disconnection, and the first few cycles of the run down period.

\section{CONCLUSIONS}

This paper investigates the detection of broken rotor bar faults using both the Fourier transform and wavelet analysis of the rundown induced voltage in induction motors. The motors were tested under different levels of broken rotor bar fault and under different loading conditions, varying from no load to full load.

The Fourier transform results showed changes in the harmonic components of the induced voltage as the fault severity increased. In particular the $5^{\text {th }}, 13^{\text {th }}$, and $17^{\text {th }}$ harmonics showed significant variations. However the results did not show consistent trends with increasing severity of fault, and load variations had a significant effect, making it difficult to interpret the results.

The proposed wavelet approach was based on the broken rotor bars causing changes in the motor torque for a given slip. This gave reasonable results, especially with higher fault severities when the motor was operating at higher loads. The technique however does have some significant limitations when applied to practical systems as it requires tests be

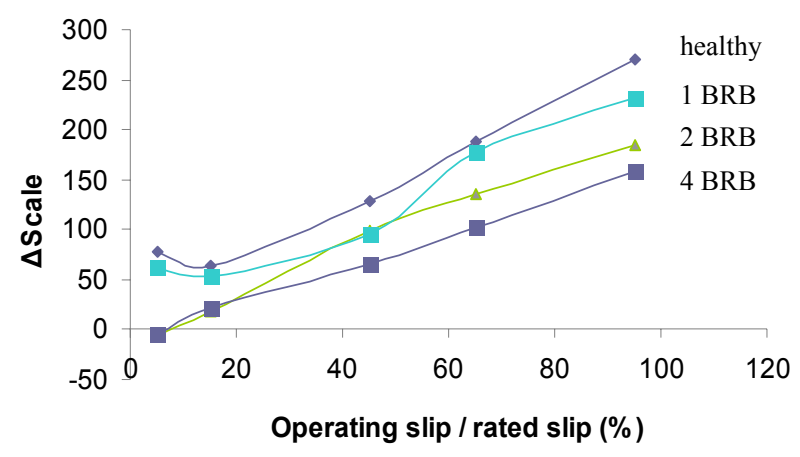

Fig. $7-\Delta$ (wavelet scale) of the rundown induced voltage.

Fig. $8-\Delta$ (wavelet scale) of the rundown induced voltage with more

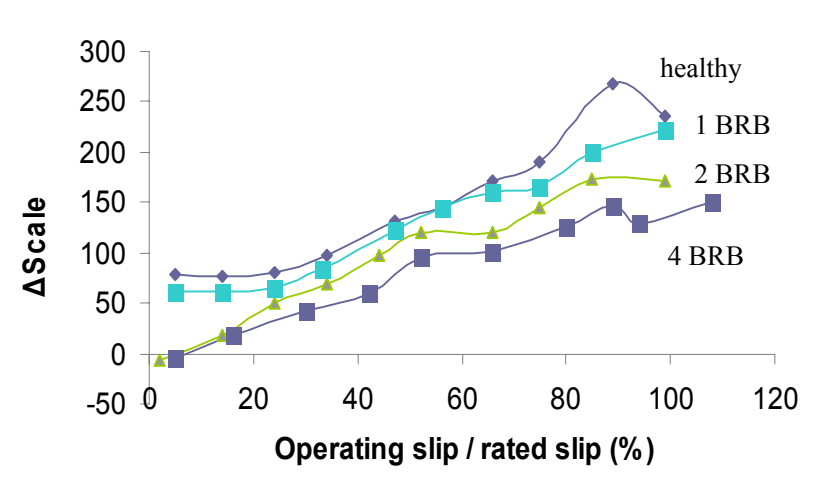

Foad levels from a second test.
lo $8-\Delta($ wavelet carried out with the motor in the healthy state to develop a baseline response, and is also sensitive to changes in load, system inertia, rotor temperature and supply voltage.

\section{REFERENCES}

[1] A. Bellini, F. Filippetti, G. Franceschini, C. Tassoni, and G. B. Kliman, "Quantitative evaluation of induction motor broken bars by means of electrical signature analysis," IEEE Trans. on Industry Applications, vol. 37, pp. 1248-55, 2001.

[2] M. E. H. Benbouzid, "A Review of Induction Motor Signature Analysis as a Medium for Faults Detection," IEEE Trans. on Industrial Electronics, vol. 47, no. 5, pp. 984-993, Oct. 2000.

[3] J. Siau, A. Graff, W. L. Soong, and N. Ertugrul, "Broken Bar Detection in Induction Motors Using Current and Flux Spectral Analysis," Australian Journal of Electrical and Electronic Engineering, vol. 1, no. 3, pp. 171-177, 2004.

[4] R. Supangat, N. Ertugrul, W. L. Soong, D. A. Gray, C. Hansen, and J. Grieger, "Broken Rotor Bar Fault Detection in Induction Motors Using Starting Current Analysis," IEEE 11th European Conference on Power Electronics and Applications, Dresden, Germany, Sept. 2005.

[5] H. Douglas, P. Pillay, and A. K. Ziarani, "A New Algorithm for Transient Motor Current Signature Analysis Using Wavelets," IEEE Trans. on Industry Applications, vol. 40, no. 5, pp. 13611368, Sep./Oct. 2004.

[6] Z. Zhang, Z. Ren, and W. Huang, "A novel detection method of motor broken rotor bars based on wavelet ridge," IEEE Trans. on Energy Conversion, vol. 18, no. 3, pp. 417-423, 2003.

[7] J. Milimonfared, H. M. Kelk, S. Nandi, A. D. Minassians, and H. A. Toliyat, "A Novel Approach for Broken Rotor Bar Detection in Cage Induction Motors," IEEE Trans. on Industry Applications, vol. 35, no. 5, pp. 1000-1006, Sept.-Oct. 1999.

[8] F. Cupertino, E. de Vanna, L. Salvatore, and S. Stasi, "Analysis Techniques for Detection of IM Broken Rotor Bars After Supply Disconnection," IEEE Trans. on Industry Applications, vol. 40, no. 2, March/April 2004.

[9] I. Daubechies, "The wavelet transform, time-frequency localization and signal analysis," IEEE Trans. on Information Theory, vol. 36, pp. 961-1005, 1990. 